\title{
Analyse multicritère et SIG pour faciliter la concertation en aménagement du territoire : vers une amélioration du processus décisionnel? \\ Promoting Regional Planning Dialogue Through Multi-Criteria Analysis and GIS: Improving the Decision-Making Process
}

\section{Carlo Prévil, Marius Thériault et Joël Rouffignat}

Volume 47, numéro 130, avril 2003

URI : https://id.erudit.org/iderudit/007968ar

DOI : https://doi.org/10.7202/007968ar

Aller au sommaire du numéro

Éditeur(s)

Département de géographie

ISSN

0007-9766 (imprimé)

1708-8968 (numérique)

Découvrir la revue

Citer cet article

Prévil, C., Thériault, M. \& Rouffignat, J. (2003). Analyse multicritère et SIG pour faciliter la concertation en aménagement du territoire : vers une amélioration du processus décisionnel? Cahiers de géographie du Québec, 47(130), 35-61. https://doi.org/10.7202/007968ar
Résumé de l'article

La planification d'une section de parc linéaire de moins de 15 kilomètres a généré une situation très complexe à la MRC de Portneuf (Québec, Canada). La MRC, face au mécontentement de certains groupes de citoyens, a eu à définir une ligne d'action à partir de cinq scénarios d'aménagement produits par les différents groupes de parties prenantes. Les consultations n'ayant pas abouti, les protagonistes ont poursuivi le débat devant les tribunaux. Dans cet article issu des travaux de notre thèse de doctorat, nous voulons montrer comment l'application d'une approche méthodologique intégrant un référentiel d'aménagement, des outils de systèmes d'information géographique (SIG) et une méthode d'analyse multicritère à la décision (AMCD) pourrait aider à départager les préférences des acteurs territoriaux pour faciliter la négociation et simplifier le processus décisionnel. 


\title{
Analyse multicritère et SIG pour faciliter la concertation en aménagement du territoire : vers une amélioration du processus décisionnel?
}

\author{
Carlo Prévil ${ }^{1}$, Marius Thériault ${ }^{2}$ et Joël Rouffignat ${ }^{3}$ \\ ${ }^{1}$ Groupe d'études interdisciplinaires en géographie et environnement régional \\ (GEIGER), Département de géographie, Université du Québec à Montréal \\ 2 Département d'aménagement et CRAD, Université Laval \\ ${ }^{3}$ Département de géographie, Université Laval \\ previl.carlo@uqam.ca \\ Marius.Theriault@crad.ulaval.ca \\ Joel.Rouffignat@ggr.ulaval.ca
}

\section{Résumé}

La planification d'une section de parc linéaire de moins de 15 kilomètres a généré une situation très complexe à la MRC de Portneuf (Québec, Canada). La MRC, face au mécontentement de certains groupes de citoyens, a eu à définir une ligne d'action à partir de cinq scénarios d'aménagement produits par les différents groupes de parties prenantes. Les consultations n'ayant pas abouti, les protagonistes ont poursuivi le débat devant les tribunaux. Dans cet article issu des travaux de notre thèse de doctorat, nous voulons montrer comment l'application d'une approche méthodologique intégrant un référentiel d'aménagement, des outils de systèmes d'information géographique (SIG) et une méthode d'analyse multicritère à la décision $(\mathrm{AMCD})$ pourrait aider à départager les préférences des acteurs territoriaux pour faciliter la négociation et simplifier le processus décisionnel.

Mots-clés : aménagement du territoire, environnement, systèmes d'information géographique (SIG), aide multicritère à la décision (AMCD), négociation territoriale.

\begin{abstract}
Promoting Regional Planning Dialogue Through Multi-Criteria Analysis and GIS: Improving the Decision-Making Process

Planning a section of a 15-km linear park has generated a delicate situation in the regional county municipality of Portneuf (Quebec, Canada). Faced with discontent among some citizens groups, the county had to define a course of action based on five management designs proposed by the different groups of promoters involved. Since consultations resulted in an impasse, opponents have taken the case before the courts. This paper, based on our doctorate thesis work, attempts to demonstrate how a well-balanced methodological approach that incorporates a planning system of reference, geographic information system (GIS) tools and multi-criteria decision analysis (MCDA) can sometimes help to set apart the preferences of regional players to promote negotiations and simplify the decisionmaking process.
\end{abstract}

Key Words: urban and regional planning, environmental studies, geographic information system (GIS), multi-criteria decision analysis (MCDA), planning process, community participation. 


\section{INTRODUCTION}

L'aménagement du territoire vise essentiellement à améliorer l'équilibre fonctionnel des espaces régionaux par rapport aux revendications des acteurs territoriaux (Brunet, 1997). Au Québec, le processus décisionnel régional est assujetti à un cadre juridique qui définit le contexte décisionnel (Loi 125 sur l'aménagement et l'urbanisme). Il est complexifié en raison de la multiplicité des données, des acteurs et des intérêts à prendre en compte à chaque phase de l'élaboration des choix (Caron, 1995). Dans les situations où les aménagistes doivent définir leur proposition d'aménagement en tenant compte de points de vue différents, à la limite contradictoires, il convient d'admettre la faiblesse des approches méthodologiques utilisées par les acteurs territoriaux (Guay, 2001). Les éléments de référence utilisés sont généralement insuffisants pour permettre aux aménagistes et aux décideurs :

- de gérer l'ensemble des interrelations connues dans les processus décisionnels (Schärlig, 1985; Simos, 1990);

- de tenir compte de la complexité de l'aménagement du territoire dans la perspective du développement durable (Jacobs et Sadler, 1990);

- de répondre à la demande sociale d'information sur l'environnement afin de rehausser la participation publique (CMED, 1992); et,

- de rechercher les consensus territoriaux facilitant le processus décisionnel (Nijkamp et al., 1990; Fisher et Nijkamp, 1993; Vodoz, 1994).

Cette situation est exacerbée par les transferts de responsabilité vers les gouvernements régionaux et l'impératif de la prise en compte des préoccupations environnementales (Waaub, 1991; Proulx, 1998). Ces enjeux contemporains nous invitent à élaborer et à expérimenter des approches méthodologiques visant à améliorer les procédures en aménagement du territoire de manière à mieux intégrer les facteurs de l'évaluation environnementale et à renforcer la démocratie participative afin de maximiser la satisfaction du public et de promouvoir le développement durable (Carver, 1991).

L'aménagement du territoire se caractérise surtout par ses dimensions politiques, car il vise d'abord la concertation et la justice sociale. Cependant, la dimension technique a souvent constitué sa pierre d'achoppement dans la pratique. On peut citer Guay (2001 : 141) quand il affirme : «Et si la technique sans la théorie est aveugle, la théorie sans la technique reste impuissante », ce qui conduit à la motivation fondamentale de notre travail : la conservation du sol, comme ressource naturelle rare, et sa gestion, comme produit social, nécessitent des méthodes et des outils, autant pour favoriser l'arbitrage et l'émergence de pactes sociaux sur son affectation que pour veiller à sa pérennisation dans une vision de développement durable.

Selon Schärlig (1985), on peut classer les problématiques d'aménagement parmi ces « décisions de gestion qu'on ne peut pas isoler de leur contexte ». En effet,

- elles renvoient souvent à la problématique du choix ou de la mise en rang, avec un contenu politique qui peut nécessiter la négociation (Roy et Bouyssou, 1993); 
- elles sont multidimensionnelles en conjuguant des aspects biophysiques et socio-économiques, les valeurs, les traditions et les rapports de force à l'œuvre dans le milieu (Malczewski, 1999);

- le territoire étant un construit des acteurs locaux (Grasland, 1994), l'évaluation de la plupart de ses composantes varie en importance selon le groupe-cible, ce qui rend parfois incomparables certains scénarios d'intervention (Martin et al., 1999). Son appréciation est ainsi fortement empreinte de valeurs et conséquemment porteuse de germes de discordes ou de conflits (Vincke, 1989); et

- la limite et la portée des actions sont souvent difficiles à cerner, elles sont semi-structurées et s'accommodent rarement d'une démarche classique de recherche opérationnelle (Schärlig, 1985).

\section{ENJEUX D’UNE MÉTHODE D'AIDE À LA DÉCISION EN AMÉNAGEMENT DU TERRITOIRE}

Lorsque l'espace intervient dans le déroulement d'une activité, non seulement comme support passif, mais aussi comme facteur actif de sa propre transformation, on ne peut plus le considérer uniquement comme une structure stable à décrire, à expliquer ou à synthétiser. Il importe alors de l'analyser (analyse spatiale) et d'anticiper son évolution en fonction du couple : attribution-localisation (Openshaw et Openshaw, 1997). L'espace et le temps peuvent être considérés en continu (durée, étendue); on mesure alors des intensités ou les densités du phénomène (concentration résidentielle, débit de circulation, profondeur de vue). Si elles sont discrétisées (événements, période, lieu, zone), on caractérise des états différents (piste cyclable en été et piste de motoneiges en hiver) ${ }^{1}$. On aboutit alors dans l'univers de la modélisation systémique, qui constitue, selon Thériault et Claramunt (1999), le domaine d'application par excellence de l'analyse spatiale et des systèmes d'information géographique (SIG).

En planification territoriale, on est souvent amené à construire et à évaluer des scénarios d'intervention en tenant compte de multiples utilisations possibles des ressources, souvent dans un contexte d'incertitude (Fisher et Nijkamp, 1993; Hickey et Jankowski, 1997). Les informations relatives à l'état et à la dynamique de systèmes territoriaux, comme les régions, sont de natures diverses (échelles, temps, dépendances, valeurs, croyances et objectifs), en plus d'être destinées à une grande diversité de parties prenantes (Prévil, 2000). Pour être pertinentes, les approches aménagistes doivent considérer davantage l'objet du processus décisionnel et la faisabilité des consensus, notamment en mettant en contexte les facteurs structurant la décision afin d'éviter les confrontations et les débats politisés à outrance. L'élaboration des choix peut être largement facilitée par le recours à des méthodes permettant de comparer, par analyse multicritère de décision $(A M C D)$, les conséquences des interventions et d'anticiper l'emprise territoriale des potentiels, des contraintes du milieu (SIG) autant que l'effet des affectations, des préférences, des désirs et des aspirations propres à diverses parties prenantes ayant des intérêts distincts (SIG et $A M C D$ ). 


\section{POUR UNE DÉMARCHE COMMUNICATIONNELLE EN AMÉNAGEMENT DU TERRITOIRE}

La formalisation des perceptions propres à chacun des groupes d'acteurs territoriaux contribue à la constitution d'une base de connaissance sur le territoire. Le partage de cette connaissance par les parties peut favoriser une évolution dans l'intelligence d'ensemble d'une problématique territoriale grâce à une systématisation de l'information géographique factuelle et perçue, et ceci, à toutes les phases du processus décisionnel. Hanna (2000) évoque les avantages particuliers résultant de la volonté politique de favoriser la participation et l'enrichissement collectif relié à l'implantation de ces aspects communicationnels et informationnels dans l'aménagement du territoire. L'information s'impose alors comme un élément décisionnel de premier ordre et l'information environnementale s'inscrit comme le garant de la pertinence des décisions se réclamant des idéaux de bien-être, de justice sociale, d'équité inter-générationnelle et de développement durable.

Ainsi, dans les situations où la préférence n'est pas claire entre différentes affectations du sol ou divers scénarios d'aménagement, une approche méthodologique basée sur un couplage de SIG et d'AMCD permet de modéliser les aspirations des parties en fonction de critères choisis et pondérés par ces derniers, afin de négocier l'affectation du sol (Laaribi, 2000). Dans cet article, notre objectif principal consiste à présenter un exemple d'application combinant les SIG et l'AMCD pour comparer des scénarios d'aménagement. Cette étude s'inscrit dans le courant des recherches portant sur la modélisation des processus décisionnels territoriaux (Rosenhead, 1989; Malczewski, 1999).

L'approche combine des méthodes de géographie et des notions en aménagement du territoire et en études environnementales afin de satisfaire la demande sociale d'information sur l'environnement et de faciliter la participation des acteurs territoriaux (avec la diversité de leurs revendications) comme principe de bonne gouvernance et de réalisation des objectifs de l'Agenda 21. Il s'agit de favoriser la démarche de prise de décision par consensus telle que la définit Vodoz (1994) comme une forme de négociation intégrative, itérative et coopérative. Une telle entreprise requiert toutefois des méthodologies et des outils permettant de gérer et d'analyser le continuum informationnel (connaissance - décision) qui conditionne l'espace social et l'évolution du territoire (Joliveau et Etlicher, 1998; Goodchild, 2000).

\section{APPORT DES SIG À LA PLANIFICATION TERRITORIALE STRATÉGIQUE}

La planification stratégique constitue une fonction fondamentale de la gestion territoriale qui comporte trois étapes fondamentales, soit la détermination des objectifs, l'élaboration des scénarios d'action ou de réglementation et le choix du scénario à réaliser.

Il importe alors d'établir la distinction entre deux notions déjà évoquées dans ce texte, à savoir : la décision et le choix. La décision est un acte d'autorité déterminant ce qui doit être fait ou réalisé alors que le choix est une activité technique permettant de distinguer les scénarios d'action retenus, les uns des autres (Simon, 1977; Buogo et Chevallier, 1995). On comprend donc que la décision sera d'autant 
moins arbitraire et plus facile à prendre que les travaux relatifs aux choix auront été approfondis et que l'information relative à la décision aura été bien spécifiée, clairement énoncée et précisément mesurée.

Les SIG permettent le traitement, la gestion, l'analyse, l'intégration et la modélisation des données géographiques ainsi que des processus qui transforment le territoire. Ils peuvent gérer les informations intégrées, à dimensions multiples, représentant des milieux complexes, en plus de pouvoir modéliser des scénarios d'aménagement. Ils aident à hiérarchiser les facteurs à considérer pour la résolution des problèmes en révélant notamment l'intensité autant que la qualité des interdépendances entre les lieux en fonction de la distance géographique, économique, temporelle, culturelle ou sociale qui les sépare (Openshaw et Openshaw, 1997; Goodchild, 2000; Burrough et Mc Donnel, 1998).

Toutefois, il reste encore du chemin à parcourir avant de produire des représentations du territoire bien comprises et acceptées de l'ensemble de la population et des informations pleinement adaptées aux différents enjeux et groupes d'utilisateurs impliqués dans la prise de décision en aménagement du territoire (Thériault, 1996). La démarche de prise de décision requiert des moyens complémentaires pour comparer (choix, tri ou mise en rang) plusieurs scénarios d'aménagement.

C'est le domaine d'action par excellence des méthodes d'analyse multicritère (Malczewski, 1999). Grâce à un traitement explicite des données, des critères et des préférences, ces dernières favorisent la transparence, la communication, la concertation et l'implication de toutes les parties (Debrincat et Meyère, 1998; Prévil, $2000)^{2}$. Ainsi, en aménagement régional, les idéaux associés au développement durable pourront mieux émerger grâce à l'expérimentation d'approches nouvelles facilitant une démarche consensuelle telle que la définit Vodoz (1994) : « la création d'une solution inédite, susceptible de satisfaire les partenaires de la décision de manière plus profonde que suite à une série de concessions ».

\section{COMPLÉMENTARITÉ DES SIG ET DE L'AMCD}

L'analyse multicritère repose sur un ensemble de procédures permettant de détailler un problème décisionnel portant sur des situations complexes. Dans l'analyse multicritère, on cherche un domaine de résolution pouvant tenir compte de l'ensemble des critères susceptibles d'influencer la décision. Le critère se définit comme un facteur à prendre en considération pour évaluer un scénario donné ou pour apprécier une occasion d'action (Laaribi, 2000; Hickey et Jankowski, 1997).

Les principes de l'AMCD témoignent autant d'une évolution des pratiques des utilisateurs que d'un changement profond dans les modalités du processus décisionnel (Roy et Bouyssou, 1993; Debrincat et Meyère, 1998). L'AMCD permet de composer avec la multiplicité, la divergence et la nature (quantitative ou qualitative) des critères en vue d'aboutir à des compromis acceptables (Simos, 1990).

Sur le plan opérationnel, l'AMCD compare des scénarios d'actions ou des variantes en fonction de problématiques générales, définies par Roy (1985 : 74) : 
- de choix, quand le résultat recherché découle d'une procédure de sélection; par exemple, pour établir une priorité entre un projet de bibliothèque, un centre sportif, un parc et une piste cyclable;

- de tri, pour une procédure d'affectation; par exemple, pour catégoriser les différents lots de la zone verte (réservée pour l'agriculture) d'une MRC afin de sélectionner ceux qui seraient propices à l'établissement d'un élevage porcin;

- de classement, pour établir des classes de priorité; par exemple, pour classer divers scénarios d'intervention du moins au plus intéressant; et,

- de description, pour améliorer la compréhension d'ensemble de différentes actions afin de mettre en contexte les conséquences possibles des interventions.

Il existe différentes méthodes d'AMCD, chacune proposant des modalités particulières. Elles se différencient surtout en fonction des arbres de décision utilisés pour définir les ensembles de solution (Maystre et al., 1994). Dans le contexte des gouvernements régionaux, le choix d'une méthode par rapport à une décision d'aménagement donnée se fait en tenant compte (Nijkamp et al., 1990) du type de problématique étudiée; des caractéristiques de la base de connaissance sur le territoire, du système d'information disponible et des données traitées (biophysiques, socio-économiques); du mode de représentation et d'évaluation des phénomènes étudiés et de la limite ou de la portée prévue des actions étudiées.

Figure 1 Principales étapes de la démarche de planification retenue. Tracé de la piste cyclable, MRC de Portneuf, 1996

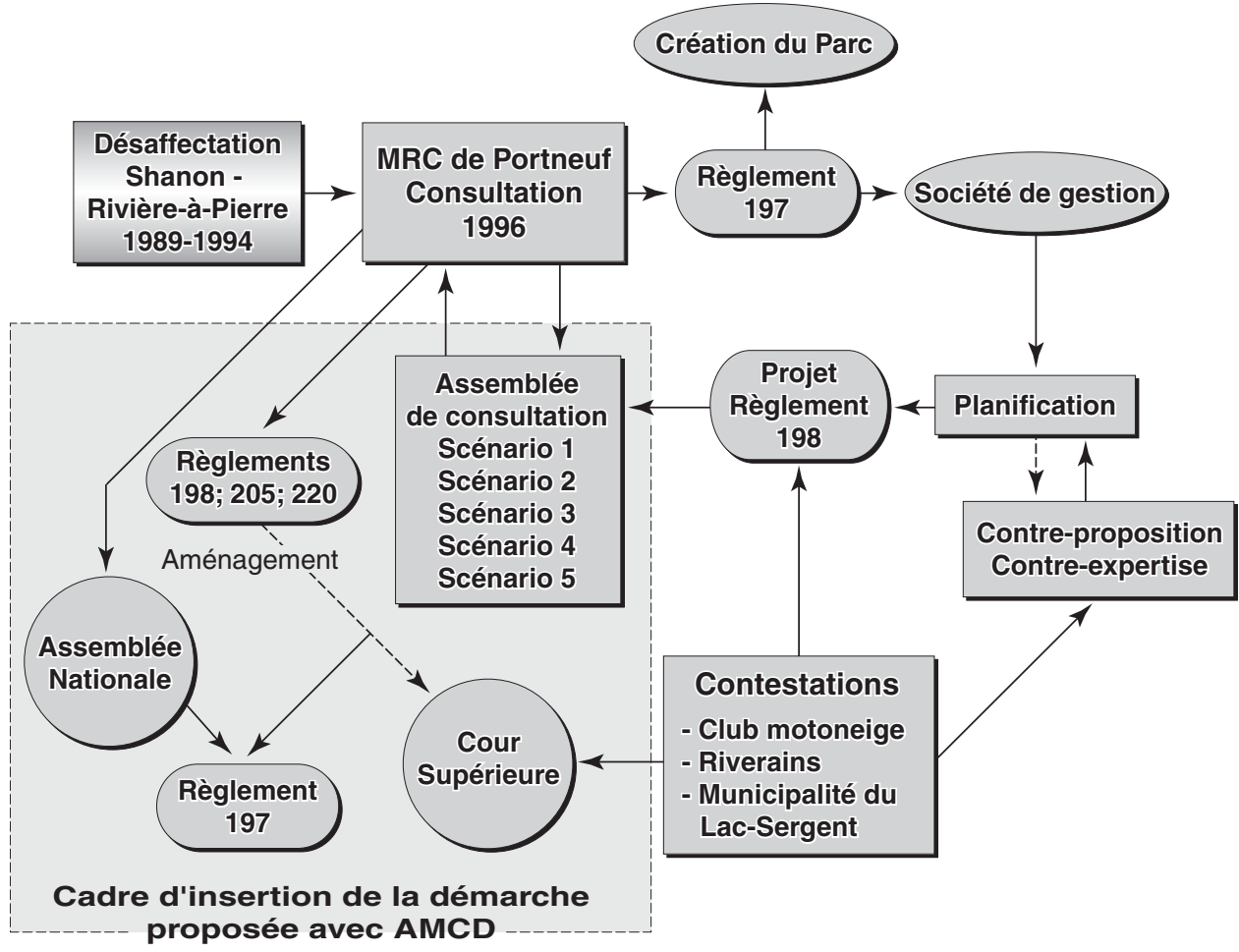


Figure 2 Tracé de la piste cyclable, MRC de Portneuf, 1996

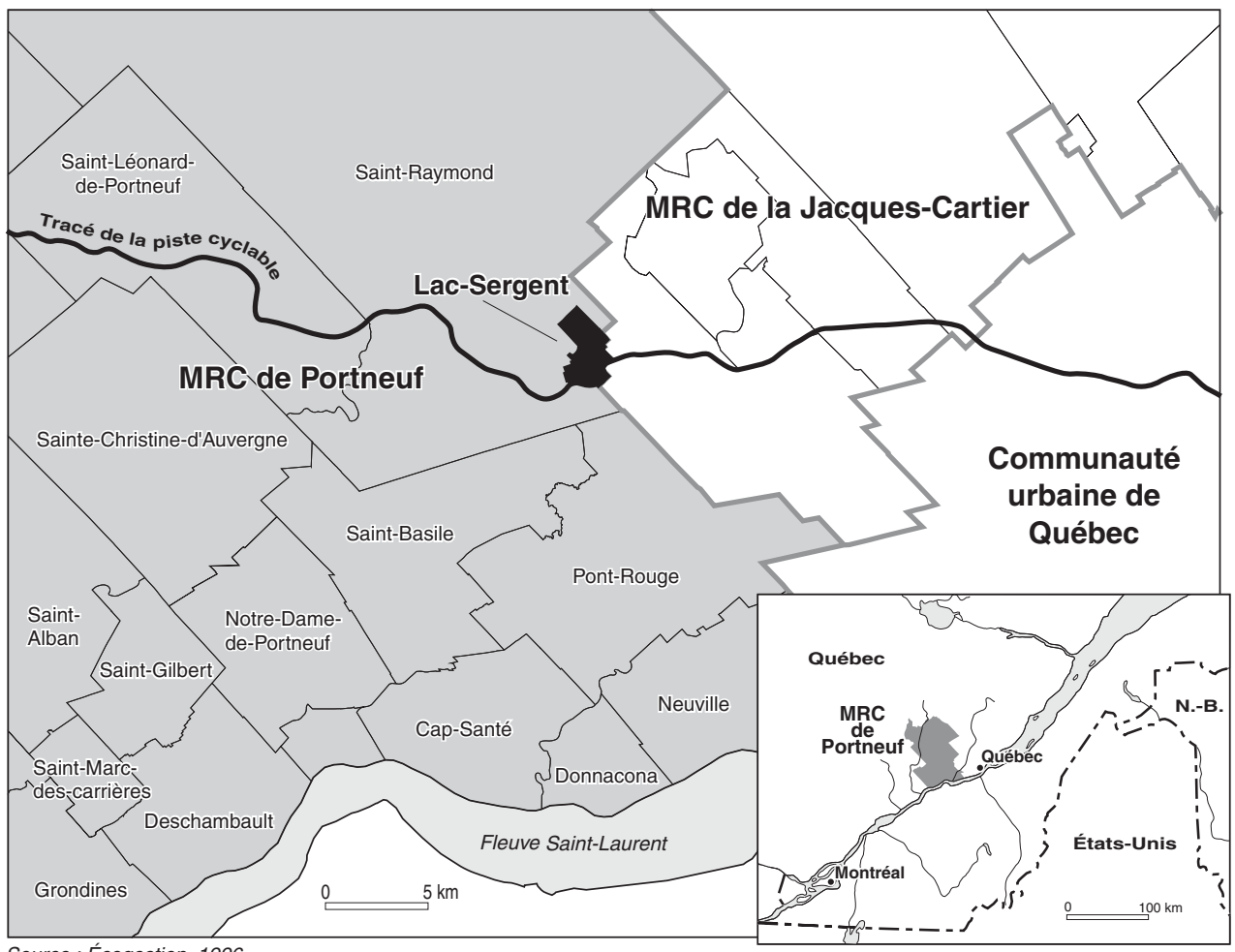

Source : Écogestion, 1996

\section{APPLICATION DES SIG ET DE L'AMCD POUR L'AMÉNAGEMENT D'UNE PISTE CYCLABLE DANS LA MRC DE PORTNEUF}

En 1989 et 1993, le Canadien National a obtenu de l'Office national des transports du Canada l'autorisation d'abandonner les corridors ferroviaires desservant les liaisons Saint-Raymond - Rivière-à-Pierre et Shannon - Saint-Raymond, dans les MRC de La-Jacques-Cartier et de Portneuf. Àl'initiative des citoyens de ces régions (pétition de plus de 7500 signatures), ces MRC ont décidé de réaffecter ce corridor, long de 63 kilomètres, en un parc régional linéaire créé à des fins récréatives et touristiques et s'intégrant dans le circuit de la Route verte du Québec ${ }^{3}$. À la suite d'ententes avec des partenaires attitrés ${ }^{4}$ et d'une étude de faisabilité, les deux MRC ont décidé de mettre sur pied un organisme à but non lucratif, la Société de la piste Jacques-Cartier/Portneuf, chargé de réaliser, d'aménager et de gérer ce parc prenant la forme d'un corridor multifonctionnel (figure 1).

La vocation de " piste cyclable » lui a été attribuée pour la période estivale et celle de "piste de motoneige » pour l'hiver. Le concept retenu a été décomposé en trois sections correspondant à trois étapes de planification et d'aménagement [Shannon/Duchesnay $(11 \mathrm{~km})$, Duchesnay/Saint-Raymond $(18 \mathrm{~km})$ et SaintRaymond/Rivière-à-Pierre $(34 \mathrm{~km})]$. À la MRC de Portneuf, le corridor traverse les municipalités de Lac-Sergent, de Saint-Raymond, de Saint-Léonard de-Portneuf et de Rivière-à-Pierre (figure 2). 
Le 20 mars 1996, le Conseil de la MRC a voté le règlement 197 déterminant l'emplacement du parc régional linéaire. À partir de cette date, le processus d'aménagement a commencé à essuyer ses premiers revers. Le 26 juin 1996, la municipalité du Lac-Sergent a déposé un avis pour faire part de ses objections et au cours du même mois, des villégiateurs de cette même municipalité (Zone $H$ ) ont publié une contre-proposition portant sur un tronçon de la deuxième section, à laquelle s'ajoutaient quelques critiques sur le concept d'ensemble. Devant cette opposition, la MRC a commandé une expertise auprès d'une firme d'ingénieurs (BPR) et créé une Commission d'aménagement (Loi 125, art. 53.1) chargée de mener des consultations publiques et de dégager un " consensus " pour l'aménagement de ce tronçon conflictuel. Deux cent cinquante personnes ${ }^{5}$ ont assisté à l'assemblée de consultation (juillet 1996) et 5 mémoires y ont été déposés. Le 19 février 1997, la Commission a déposé un projet de règlement amendant le précédent sur la base des opinions, mémoires et études complémentaires présentés lors de l'assemblée de consultation. La municipalité du Lac-Sergent a fait part de ses nouvelles critiques le 31 mars 1997. Le règlement 198 visant la planification du règlement multifonctionnel sur l'ancien corridor ferroviaire a été voté le 15 mai 1997. La municipalité du Lac-Sergent s'est opposée à ce règlement. Les consultations n'ayant pas abouti, les protagonistes ont choisi de poursuivre le débat devant les tribunaux ${ }^{6}$.

\section{LE CONTEXTE DÉCISIONNEL DANS LA MRC ET L'ÉLABORATION DE SCÉNARIOS}

Le projet de piste cyclable relève du champ de compétence de la MRC visant à fournir des services à l'ensemble des municipalités ou à certaines d'entre elles. Pour cette problématique d'aménagement de corridor multifonctionnel, l'univers décisionnel était organisé autour des intérêt suivants :

- Les attentes économiques, les perspectives régionales et environnementales exprimées par la MRC de Portneuf, la Société de la piste, le Service d'aménagement de la MRC et d'autres groupes d'intérêt des régions voisines.

- Les préoccupations individuelles, locales et écologiques exprimées par les citoyens de la Zone-H, les autres résidents riverains, la municipalité du Lac-Sergent et leurs alliés provenant d'autres municipalités de villégiature.

- Les perspectives régionales de loisirs exprimées par les clubs de motoneigistes (local et régional) qui s'identifient comme des amants de la nature sauvage.

Cet exemple d'application se base essentiellement sur les informations et les documents publics analysés dans le contexte de cette consultation et sur des entrevues avec quelques intervenants bien informés du déroulement du processus décisionnel. Comme le conflit était encore pendant au moment où ce travail a été réalisé, il n'était pas possible d'inviter tous les acteurs afin de réaliser une simulation les impliquant. Dans cette application, nous voulons montrer comment une approche basée sur la représentation géomatique du territoire (SIG) et sur l'AMCD aurait pu contribuer à clarifier les préférences des groupes d'acteurs et à faciliter le déroulement du processus décisionnel. 
Le concept et les objectifs du parc linéaire ont été acceptés, par consensus, par les populations des deux MRC impliquées. Toutefois, les dissensions sont apparues lorsque les détails de la réalisation du projet ont été rendus publics. Même si ce projet comportait aussi des dimensions biophysiques, sociopolitiques et économiques, ce sont principalement des considérations d'ordre social et environnemental qui ont été soulevées lors de la consultation portant sur le tronçon de Lac-Sergent.

Le tronçon conflictuel est long de moins de 4 kilomètres et est compris entre le lac Sergent et un ensemble de plus de 75 maisons de villégiature (figure 3). Le tableau 1 présente succinctement les cinq scénarios découlant de cet ensemble d'interactions et à partir desquels la Commission d'aménagement a formulé le Projet de règlement 198, en février 1998. Ces scénarios représentent cinq variantes d'aménagement avec des implications associées aux préoccupations soulevées par les parties. Les scénarios sont présentés sommairement, dans le tableau 1 et la figure 3, avec une estimation des coûts de réalisation.

Tableau 1 Présentation des cinq scénarios d'aménagement du tracé de la piste cyclable. Municipalité du Lac-Sergent, 1996

\begin{tabular}{|c|c|c|c|c|c|}
\hline & Scénario 1 & Scénario 2 & Scénario 3 & Scénario 4 & Scénario 5 \\
\hline Promoteurs & $\begin{array}{l}\text { Conseil régional } \\
\text { des loisirs (CRL) }\end{array}$ & $\begin{array}{c}\text { Commission } \\
\text { d'aménagement }\end{array}$ & $\begin{array}{c}\text { Citoyens de } \\
\text { la zone } \mathrm{H}\end{array}$ & Firme BPR & $\begin{array}{l}\text { Commission } \\
\text { d'aménagement } \\
\text { etCRL }\end{array}$ \\
\hline Principe & $\begin{array}{l}\text { Ancien corridor } \\
\text { ferroviaire }\end{array}$ & $\begin{array}{l}\text { Ancien corridor et } \\
\text { contournement pour } \\
\text { les mononeiges }\end{array}$ & $\begin{array}{c}\text { Chaussée désignée } \\
\text { par la Route Tour- } \\
\text { du-lac-sud }\end{array}$ & $\begin{array}{l}\text { Contournement local } \\
\text { par la Côte-à-Pagé }\end{array}$ & $\begin{array}{l}\text { Aménagement } \\
\text { intégral en } \\
\text { milieu forestier }\end{array}$ \\
\hline Pour & $\begin{array}{c}\text { Récupération } \\
\text { maximale, } \\
\text { piste } \\
\text { autonome, } \\
\text { contraintes } \\
\text { topographiques } \\
\text { nulles, sécurité } \\
\text { des utilisateurs }\end{array}$ & $\begin{array}{l}\text { Avantages du } \\
\text { premier scénario, } \\
\text { diminution des } \\
\text { préoccupations des } \\
\text { résidents, } \\
\text { sécurité accrue } \\
\text { pour les résidents }\end{array}$ & $\begin{array}{l}\text { Loin des sites } \\
\text { résidentiels, } \\
\text { satisfaction } \\
\text { maximale } \\
\text { des résidents }\end{array}$ & $\begin{array}{l}\text { Avantages du } \\
\text { Scénario } 3 \text { et } \\
\text { moins coûteux }\end{array}$ & Site propre \\
\hline Contre & $\begin{array}{c}\text { Opposition } \\
\text { des riverains, } \\
\text { nombreux conflits } \\
\text { à résoudre }\end{array}$ & $\begin{array}{l}\text { Opposition des } \\
\text { riverains, nombreux } \\
\text { conflits à résoudre }\end{array}$ & $\begin{array}{c}\text { Résidences de } \\
\text { Ste-Catherine } \\
\text { impliquées, } \\
\text { opposition de la } \\
\text { municipalité, } \\
\text { expropriations } \\
\text { nécessaires, risques } \\
\text { d'accidents élevés, } \\
\text { entrées privées à } \\
\text { traverser, perte } \\
\text { prononcée }\end{array}$ & $\begin{array}{c}\text { Mêmes inconvénients } \\
\text { que Scénario 3, fortes } \\
\text { pentes, parcours } \\
\text { serré, courbes } \\
\text { prononcées, réfection } \\
\text { du Tour-du-lac-sud }\end{array}$ & $\begin{array}{c}\text { Acquisition de } \\
\text { terrains en forêt, } \\
\text { négociation avec } \\
3 \text { municipalités, } \\
\text { faisabilité } \\
\text { douteuse, } \\
\text { opposition des } \\
\text { propriétaires } \\
\text { fonciers }\end{array}$ \\
\hline Longueur & $3900 \mathrm{~m}$ & non déterminée & $\begin{array}{l}1700 \mathrm{~m} \text { en forêt, } \\
400 \mathrm{~m} \text { sur chemin } \\
\text { privé et } 2100 \mathrm{~m} \text { sur } \\
\text { Tour-du-lac-sud }\end{array}$ & non déterminée & $4400 \mathrm{~m}$ \\
\hline Coûts & $248089 \$$ & $\begin{array}{c}248089 \$+ \\
\text { sentier de motoneige }\end{array}$ & $\begin{array}{c}263241 \$+ \\
\text { Tour-du-lac }+ \\
\text { sentier de motoneige }\end{array}$ & $\begin{array}{c}136180 \$+ \\
\text { Tour-du-lac-sud + } \\
\text { sentier de motoneige }\end{array}$ & $400000 \$$ \\
\hline
\end{tabular}


Figure 3 Projet de piste cyclable.

Scénarios d'aménagement des cinq meilleurs tracés.

Municipalité de Lac-Sergent, MRC de Portneuf, 1996
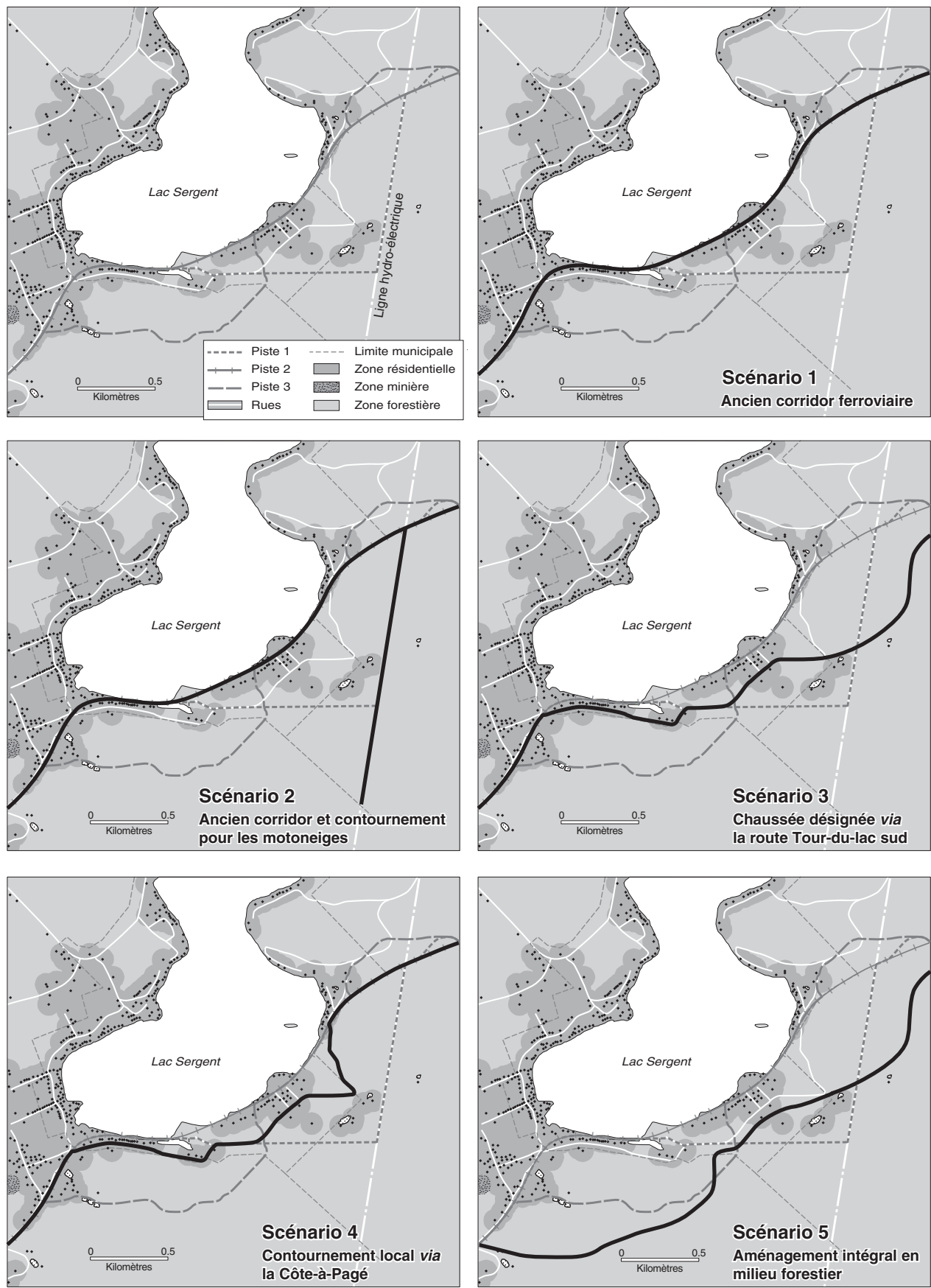

Sources : AGRÉCOR, 1998

D'après : Prévil et al., 2002 MRC de Portneuf, 1997 


\section{LA REPRÉSENTATION DU TERRITOIRE ET L'ANALYSE DES VARIANTES}

Le système de solution correspond à une thématique globale liée à la question suivante : «Comment trouver le meilleur tracé pour la piste cyclable dans le secteur du Lac-Sergent? ». En vue de répondre à cette question, nous proposons une résolution en deux phases partant des arguments soutenus par les protagonistes à l'occasion de l'assemblée de consultation.

La première phase vise à améliorer la démarche d'aménagement en ce qui concerne les considérations techniques et l'analyse coût-bénéfice. Elle examine l'utilisation des SIG pour déterminer le "tracé optimal » prenant compte simultanément des contraintes techniques et des nuisances appréhendées en rapport avec l'occupation actuelle du sol. La seconde phase approfondit les éléments de solution découlant de l'utilisation conjointe des SIG et de l'AMCD afin de mieux intégrer les dimensions sociales et politiques du processus décisionnel et de favoriser l'échange d'informations stratégiques entre les acteurs concernés.

\section{LA DÉTERMINATION DU MEILLEUR ITINÉRAIRE DE LA PISTE CYCLABLE}

Pour la conception de la piste, la Commission d'aménagement a fait une place de premier plan à certains facteurs d'aménagement se rapportant à la morphologie du terrain, à l'hydrographie, à l'emplacement du chemin de fer et des résidences en plus du réseau routier (routes, rues, ponts et chaussées) et à l'utilisation du sol. L'analyse de voisinage de ces différents facteurs permet de déterminer un tracé optimal selon un ensemble de contraintes associées aux coûts, à la sécurité, à l'efficacité et aux nuisances.

Deux options sont couramment utilisées dans les SIG pour réaliser de telles études : les méthodes vectorielles s'appuyant sur une représentation résiliaire du territoire (Thériault et al., 1999) et les méthodes matricielles, qui cherchent à déterminer la surface-coût en évaluant la friction ou l'effort nécessaire pour se déplacer sur le terrain (Eastman, 1989). La notion de distance-coût indique que le déplacement d'un point d'origine (le début du tronçon) à la destination prévue implique une impédance qui s'exprime par un coût pouvant représenter autant une opposition des occupants, une perception négative des usagers qu'une infrastructure à construire. Dans le cas qui nous intéresse, l'impédance ne se distribue pas de manière uniforme, mais est fortement conditionnée par la pente du terrain (structure anisotropique). Une fois les surfaces-coût obtenues en intégrant les types de friction considérés, on peut calculer, pour un point de destination défini, le tracé optimal ou le chemin de moindre coût, soit celui qui minimise la somme des impédances. L'exemple suivant est assez classique et vise à illustrer comment l'utilisation des SIG et de l'analyse spatiale aurait permis de déterminer le tracé optimal de la piste cyclable à Lac-Sergent.

\section{L'INTÉGRATION DES DONNÉES ET L'ANALYSE SPATIALE}

La première étape consiste à préparer les données à partir du système d'information. La carte d'utilisation du sol de la région de Portneuf a été utilisée, bien que certaines manipulations aient été rendues nécessaires pour tenir compte de l'emprise réelle au sol (objets à $2 \mathrm{D}$ ) de certains éléments linéaires (1 D). Un 
modèle numérique d'altitude (MNA) a été réalisé ainsi qu'une vue tridimensionnelle du territoire obtenue par projection et estompage (Hillshading). La carte d'utilisation du sol modifiée, les points d'origine et de fin du tronçon conflictuel, l'emprise des lignes électriques ainsi que la localisation des résidences ont été également considérés pour l'analyse spatiale. À partir de la carte d'utilisation du sol, de la position du point d'origine et de la matrice de friction liée à la pente et aux obstacles, nous avons effectué des traitements aboutissant à différentes matrices de contraintes au tracé de la piste.

En considérant la forme du terrain, le tracé de la voie ferrée et la localisation des résidences, des couloirs de visibilité ont été établis pour une hauteur de vue moyenne de 1,65 mètres. Les profondeurs de vue de 100 et 200 mètres ont été retenues pour les cyclistes. Les couloirs de visibilité pour les résidences ont été établis à des profondeurs de 100, 200 et 300 mètres. Cet aspect de la qualité de la vue a été discuté âprement lors des débats pour la délimitation des aires de voisinage et pour l'étude de structures compensatoires, comme des haies vives, pour les résidants. Les différentes matrices produites ont été ensuite exploitées pour produire les cartes d'interprétation et pour réaliser des analyses spatiales.

Dans cette analyse spatiale, chacun des facteurs a été considéré dans la mesure où il pouvait constituer une contrainte à l'accessibilité entre les deux points terminaux du tronçon. L'emprise ferroviaire a été considérée comme le lieu d'accessibilité maximale, alors que la surface des lacs a été assimilée à une contrainte insurmontable. Les autres éléments d'occupation du sol ont été pondérés, comme le montre le tableau 2 , avec un poids d'impédance (friction) exprimant leur niveau de contrainte spécifique.

Tableau 2 Facteurs complémentaires utilisés pour déterminer le tracé optimal du projet de piste cyclable. Municipalité de Lac-Sergent, 1996

\begin{tabular}{|l|c|l|}
\hline \multicolumn{1}{|c|}{ Occupation du sol } & Friction & \multicolumn{1}{c|}{ Commentaires } \\
\hline Chemin de fer & 1 & Tracé de référence \\
Lacs & 1000 & Équivaut à une barrière insurmontable pour la piste \\
Rivières & 5 & Nécessite un investissement pour construire ou élargir un pont \\
Routes & 3 & Peut nécessiter un investissement pour l'élargissement \\
Électricité & 2 & Intéressant pour le tracé existant; négociation pour les droits de passage \\
Résidence & 10 & Aires de contraintes multiples pour les nuisances \\
Mines & 6 & Aménagement particulier (trou à combler) \\
Agriculture & 5 & Valeur d'usage et occupation actuelle en restreignent l'accès \\
Forêt & 4 & Accès facile, mais entraîne des coûts d'aménagement importants \\
\hline
\end{tabular}

\section{LA VISUALISATION ET L’UTILISATION DES SIG POUR MODÉLISER LA PISTE}

Le type de traitements effectués dans le SIG permet de produire des sorties utiles pour visualiser l'insertion de la piste cyclable dans son contexte local et discuter avec la population concernée de divers aspects entourant sa réalisation et son impact sur la qualité du paysage. Les figures 3, 4 et 5 présentent quelques exemples de produits de visualisation issus du SIG. Ils permettent d'illustrer son potentiel pour améliorer la communication d'information synthétique entre les intervenants. La figure 3 présente une vue générale du territoire et les cinq 
scénarios de tracé de la piste multifonctionnelle. La figure 4 intègre la surface tridimensionnelle (estompage) drapée par certains éléments structurants du territoire comme les résidences, le lac, les routes, en plus des trois tracés résultant des traitements dans le SIG. En carton, nous avons produit des vues en perspective de chaque tracé sur la surface tridimensionnelle résultant du MNA drapé par la mosaïque photographique de la région. Cette vue de synthèse a été montée dans GEOMATICA en intégrant le MNA, une mosaïque d'orthophotos au $1: 40000$ ainsi que certaines informations résultant des analyses spatiales. Les vues en perspective (figure 5) ont été réalisées en utilisant des effets d'ombrage afin de produire des images de réalité virtuelle.

Figure 4 Tracé optimal de la piste cyclable déterminé à l'aide du SIG. Municipalité de Lac-Sergent, MRC de Portneuf, 1996

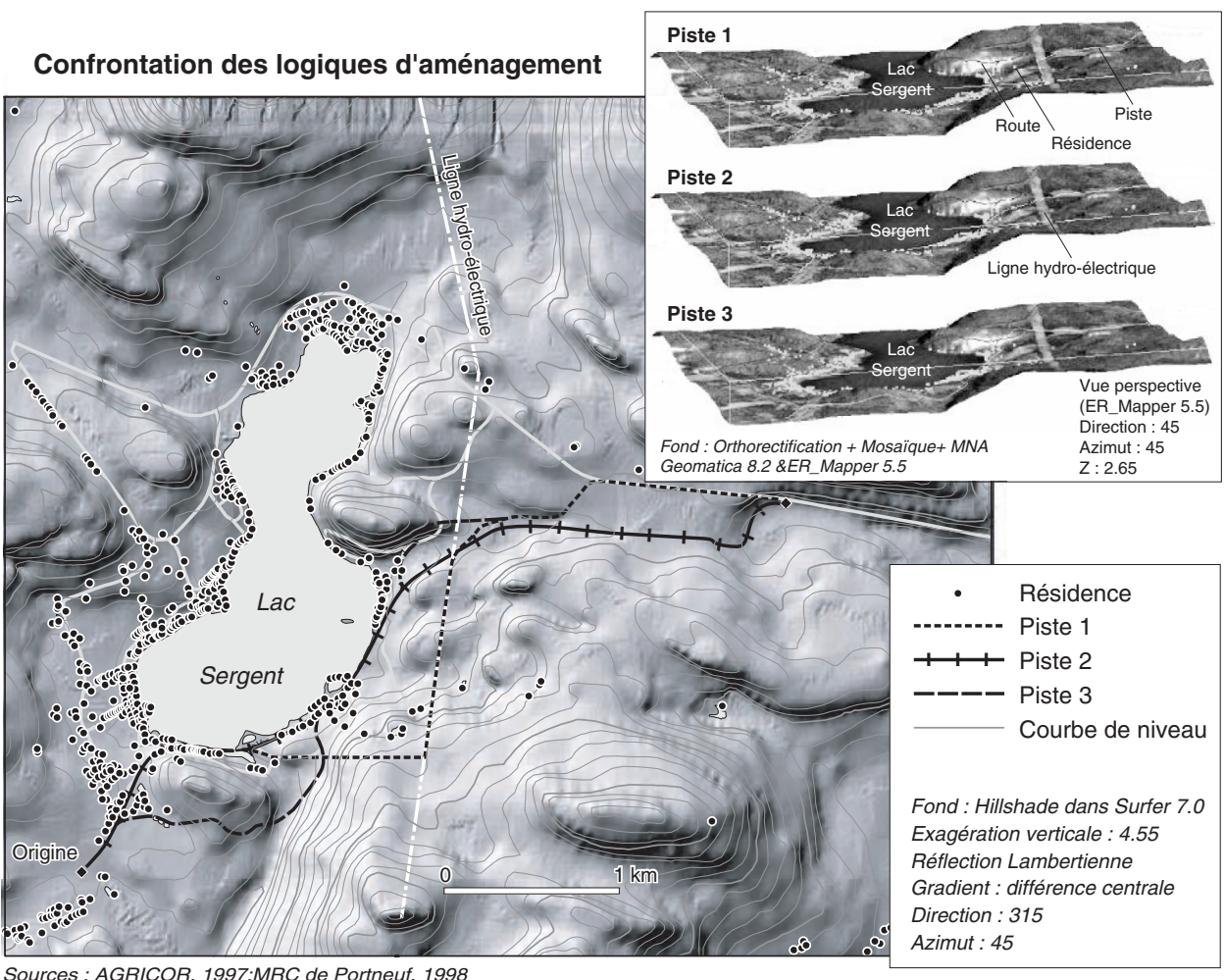

Sources : AGRICOR, 1997;MRC de Portneuf, 1998

Ainsi, diverses manipulations ont permis d'intégrer les cinq scénarios proposés au cours de l'assemblée de concertation, les trois meilleurs tracés obtenus avec les analyses spatiales réalisées par le SIG, ainsi que certains éléments structurants du territoire comme les résidences, le réseau routier, l'hydrographie et l'ancien corridor ferroviaire. Les illustrations ont été structurées ici pour une production monochrome, bien que cette dernière restreigne fortement les possibilités pour la représentation cartographique. 
Figure 5 Visualisation tridimensionnelle du projet de piste cyclable pour faciliter le processus décisionnel. Municipalité de Lac-Sergent, MRC de Portneuf, 1996

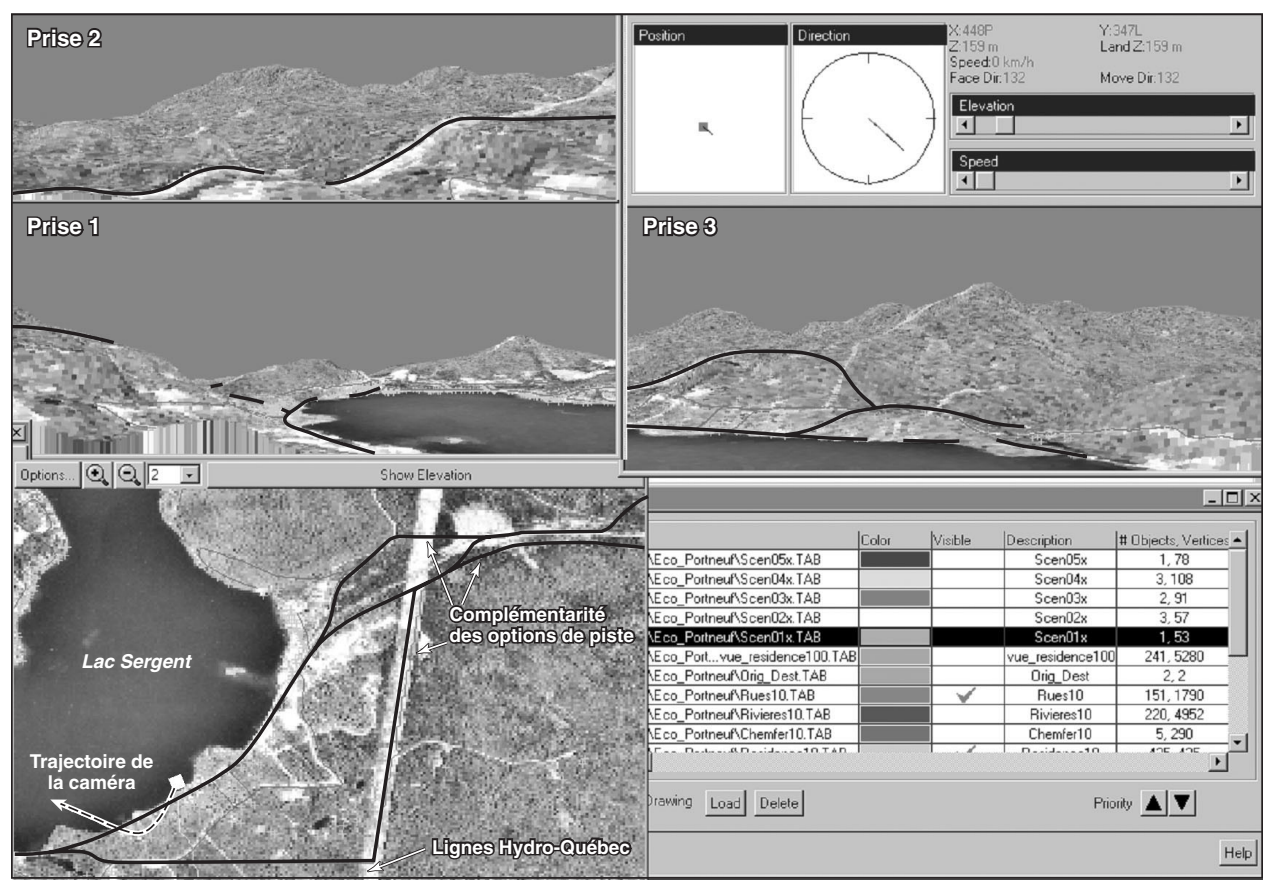

Dans ces illustrations, nous avons retenu trois tracés optimaux déterminés au moyen du SIG :

- Le tracé 1 (Costpush) respecte les contraintes liées à l'hydrographie (veto). Toutefois, dans d'autres itérations, on devrait l'associer à de plus fortes impédances au voisinage des résidences. Il recoupe peu les cinq scénarios considérés par la MRC, sauf sur les premières sections du tronçon avant l'abord du lac. Il est compatible avec les scénarios 2, 3 et 4 si on tient compte de la dérivation de la piste de motoneige.

- Le tracé 2 (Costgrow) épouse fidèlement l'ancien corridor ferroviaire. En dépit des frictions associées à la traversée des cours d'eau, il permettrait une bonne intégrité du tracé de la piste cyclable. Il reproduit les arguments mettant en valeur le corridor initial (scénario 1) et est, de ce fait, peu compatible avec le scénario 5 et, dans une certaine mesure, les scénarios 3 et 4 qui excluent les abords du lac.

- Le tracé 3 (Varcost + isotropic surface) inclut une surface isotropique dans le traitement anisotropique obtenu avec les tracés 3 et 4 . Il épouse, sur une grande longueur, le tracé du corridor ferroviaire et adopte, vers le point de départ, un tracé qui évite l'effet des fortes dénivellations aux abords des berges du lac. Incompatible avec les scénarios 3, 4 et 5, il présente néanmoins quelques similitudes avec les scénarios 1 et 2 . 
Cette présentation bien que suffisante pour la compréhension globale doit être affinée afin de permettre un aménagement effectif du site (à des fins de génie civil). En effet, elle ne tient pas compte de manière spécifique de la circulation des motoneiges et ne correspond, dans chaque cas, que de manière partielle aux différents scénarios, à l'exception des propositions pour la piste initiale avec le second tracé simulé. Une meilleure prise en charge des préoccupations soulevées concernant l'affectation des lieux requiert un approfondissement de la démarche.

Des divers résultats du SIG, se dégagent deux options pour la poursuite de l'expérimentation du processus d'aide à la décision. La première suppose un raffinement de l'utilisation du SIG qui améliorerait la représentation du territoire à plus grande échelle. Bien que pertinente pour des travaux de construction, une telle approche serait peu utile pour rehausser le débat politique et améliorer la participation citoyenne dans l'aménagement du territoire. La seconde option vise à combiner les méthodes multicritères et l'établissement des tracés à l'aide des SIG.

Dans cette première utilisation, nous avions établi une matrice géographique de friction selon un modèle combinant le relief et l'utilisation du sol. Pour approfondir cette étude, nous avons développé une méthode de représentation des valeurs et des préférences des citoyens dans le processus décisionnel de manière à rehausser la pertinence politique de la démarche.

\section{UNE MÉTHODE D'ÉVALUATION MULTICRITÈRE AVEC COMPOSANTE TERRITORIALE : L'ANALYSE DES SCÉNARIOS}

C'est cette deuxième option qui a été retenue dans le développement de cette expérience à la MRC de Portneuf. Pour ce faire, trois étapes préliminaires ont été nécessaires, à savoir : la détermination des paramètres de l'évaluation multicritère; la présentation et l'interprétation des résultats de l'évaluation multicritère; l'analyse de sensibilité.

\section{LA DÉTERMINATION DES PARAMÈTRES DE L'ÉVALUATION MULTICRITÈRE}

Dans le processus administratif, les villégiateurs et la municipalité du LacSergent ont été frustrés parce que plusieurs considérations qui leur tenaient à cœur ne semblaient pas avoir été intégrées dans l'évaluation des cinq scénarios, même si ces derniers représentaient l'ensemble des actions efficaces (Maystre, 1994). Les travaux ont porté par la suite sur trois activités essentielles à l'évaluation multicritère qui sont : la définition des critères, la standardisation de l'échelle de mesure et la pondération de ces critères.

Une analyse des documents issus de la consultation a permis d'identifier quarante-trois sujets autour desquels l'ensemble des argumentations ont été développées. Ces sujets ont été, par la suite, synthétisés en 12 critères qui représentent, en quelque sorte, l'univers des conséquences élémentaires associées à la décision d'aménagement (avec postulat d'exhaustivité et de cohérence de l'ensemble sans redondance). 
Ces critères établis, leur évaluation ou leur mesure s'obtient par l'agrégation des conséquences élémentaires mesurées à travers les 43 indicateurs élémentaires (tableau 3). Ces mesures découlent, par exemple :

- d'une évaluation monétaire (par exemple, coût de l'aménagement);

- de mesures déduites du SIG (par exemple, pourcentage de pente, nombre de pixels de faible contrainte, superficie des aires d'intervisibilité entre la piste et les résidences); ou

- d'une note attribuée selon une perception ou une anticipation par un groupe d'intervenants (par exemple, appréciation de la nuisance liée au bruit des motoneiges).

Pour un critère donné, les indicateurs sont ramenés à une même échelle et à un même axe de signification. C'est l'étape de la standardisation des valeurs.

Les parties cherchent à maximiser ou minimiser chaque critère considéré. Elles se différencient selon l'importance accordée à chaque critère en fonction de leurs convictions et de leurs valeurs. Dans une application réelle, le jeu de pondération constitue l'une des premières phases de négociation entre les parties, après l'identification des actions potentielles. Quand les décideurs n'arrivent pas à s'entendre sur la pondération des critères (ce qui est courant), il est recommandé d'effectuer l'analyse multicritère avec plusieurs jeux de poids, ce qui aide à identifier les pistes de consensus entre les groupes d'intervenants (Schärlig, 1985; Mousseau, 1993).

Pour réaliser cet exemple d'application, pour chaque critère, l'échelle de mesure (cardinale), la performance, le sens de décision ainsi que les paramètres subjectifs (indifférence, préférence et veto) ont été établis en référence aux documents de la consultation publique. Ces considérations ont guidé la spécification des valeurs consignées dans un tableau de performance. De plus, quelques entretiens semistructurés avec des membres du Service d'aménagement ayant participé au débat public nous ont aidé à définir les fourchettes de valeurs acceptables pour chaque critère.

Ces différentes étapes nécessitent également le recours à des algorithmes ou à des règles de décision selon la méthode d'agrégation multicritère retenue $\mathrm{e}^{7} \mathrm{La}$ présentation des concepts d'AMCD dans ce texte a été volontairement limitée, l'objet de la démarche actuelle consistant surtout à montrer la pertinence du couplage SIG et $\mathrm{AMCD}$ pour aider à résoudre le problème de localisation de la piste cyclable.

\section{PRÉSENTATION ET INTERPRÉTATION DES RÉSULTATS DE L'ÉVALUATION MULTICRITÈRE}

Les valeurs attribuées aux divers seuils ainsi que les notes des indicateurs ont permis d'effectuer l'évaluation initiale, selon les critères retenus. À la fin des calculs, le module Résultat du logiciel ELECTRE fournit un ensemble de matrices. Parmi les résultats obtenus, nous prendrons le temps de commenter particulièrement la matrice de concordance, la matrice des degrés de crédibilité et le graphe final (figure 6) : 
Tableau 3 Matrice d'interprétation des arguments des parties prenantes Parc linéaire régional Jacques-Cartier/Portneuf (section Lac-Sergent)

\begin{tabular}{|c|c|c|c|c|c|}
\hline Domaine & Critères & Poids & Indicateurs ou sous-critères & Proportion & Sens \\
\hline \multirow{2}{*}{ 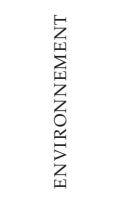 } & $\begin{array}{l}\text { Risque de pollution } \\
\text { du plan d'eau }\end{array}$ & $10 \%$ & $\begin{array}{l}\text { limitation des accès } \\
\text { détérioration du milieu aquatique } \\
\text { pollution (ampleur, nature) } \\
\text { affectation du milieu lacustre }\end{array}$ & $\begin{array}{l}25 \% \\
30 \% \\
20 \% \\
25 \%\end{array}$ & $\begin{array}{l}\min \\
\min \\
\min \\
\min \end{array}$ \\
\hline & Qualité du site & $5 \%$ & $\begin{array}{l}\text { profiter du paysage } \\
\text { apprécier l'intervisibilité }\end{array}$ & $\begin{array}{l}50 \% \\
50 \%\end{array}$ & $\begin{array}{l}\text { Max } \\
\operatorname{Max}\end{array}$ \\
\hline \multirow{3}{*}{ 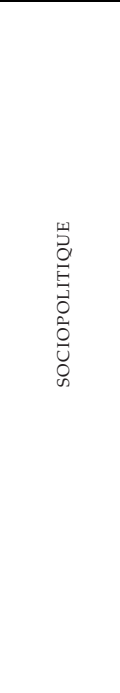 } & Quiétude des résidents & $10 \%$ & $\begin{array}{l}\text { tranquillité } \\
\text { sécurité des personnes } \\
\text { interdiction de VTT et de motos } \\
\text { accès contrôléà la piste } \\
\text { surveillance du territoire } \\
\text { préservation des droits acquis } \\
\text { intimité }\end{array}$ & $\begin{array}{r}15 \% \\
15 \% \\
15 \% \\
5 \% \\
10 \% \\
20 \% \\
20 \%\end{array}$ & $\begin{array}{l}\operatorname{Max} \\
\operatorname{Max} \\
\operatorname{Max} \\
\operatorname{Max} \\
\operatorname{Max} \\
\operatorname{Max} \\
\operatorname{Max}\end{array}$ \\
\hline & $\begin{array}{l}\text { Dévaluation des } \\
\text { propriétés riveraines } \\
\text { et hausse des coûts } \\
\text { des assurances }\end{array}$ & $10 \%$ & $\begin{array}{l}\text { nombre de délits } \\
\text { nombre de plaintes } \\
\text { nombre d'événements criminels } \\
\text { risques de délits } \\
\text { situations non réglementées } \\
\text { primes d'assurance-habitation } \\
\text { dépréciation des propriétés }\end{array}$ & $\begin{array}{l}15 \% \\
10 \% \\
10 \% \\
15 \% \\
10 \% \\
20 \% \\
20 \%\end{array}$ & $\begin{array}{l}\min \\
\min \\
\min \\
\min \\
\min \\
\text { min } \\
\text { min }\end{array}$ \\
\hline & Pouvoir local & $5 \%$ & $\begin{array}{l}\text { autonomie locale } \\
\text { cadre légal clair } \\
\text { municipalité participante } \\
\text { non contribution des riverains } \\
\text { revenus fonciers } \\
\text { retombées économiques }\end{array}$ & $\begin{array}{l}10 \% \\
10 \% \\
10 \% \\
10 \% \\
30 \% \\
30 \%\end{array}$ & $\begin{array}{l}\text { Max } \\
\operatorname{Max} \\
\operatorname{Max} \\
\operatorname{Max} \\
\operatorname{Max} \\
\operatorname{Max}\end{array}$ \\
\hline \multirow{7}{*}{ 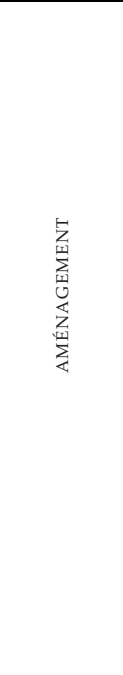 } & Récupération maximale & $10 \%$ & récupération maximale & $100 \%$ & $\operatorname{Max}$ \\
\hline & $\begin{array}{l}\text { Concentration } \\
\text { des activités }\end{array}$ & $10 \%$ & $\begin{array}{l}\text { piste autonome } \\
\text { concentration des activités }\end{array}$ & $\begin{array}{l}50 \% \\
50 \%\end{array}$ & $\begin{array}{l}\operatorname{Max} \\
\operatorname{Max}\end{array}$ \\
\hline & Topographie & $10 \%$ & pente prononcée & $100 \%$ & $\min$ \\
\hline & $\begin{array}{l}\text { Sécurité des } \\
\text { utilisateurs }\end{array}$ & $10 \%$ & $\begin{array}{l}\text { cyclistes avec automobilistes } \\
\text { risques d'accidents } \\
\text { nombre d'entrées privées }\end{array}$ & $\begin{array}{l}30 \% \\
40 \% \\
30 \%\end{array}$ & $\begin{array}{l}\min \\
\min \\
\min \end{array}$ \\
\hline & Terrains à acquérir & $5 \%$ & $\begin{array}{l}\text { nombre de résidences à négocier } \\
\text { oppposition de municipalités } \\
\text { nombre d'expropriations nécessaires } \\
\text { terrains à acheter } \\
\text { nombre de prorpiétés à morceler }\end{array}$ & $\begin{array}{l}20 \% \\
20 \% \\
20 \% \\
20 \% \\
20 \%\end{array}$ & $\begin{array}{l}\min \\
\min \\
\min \\
\min \\
\min \end{array}$ \\
\hline & $\begin{array}{l}\text { Infrastructures } \\
\text { à aménager }\end{array}$ & $5 \%$ & $\begin{array}{l}\text { réfection de chaussée } \\
\text { largeur de piste inadéquate } \\
\text { coûts du sentier de motoneiges } \\
\text { mesure d'atténuation }\end{array}$ & $\begin{array}{l}20 \% \\
20 \% \\
40 \% \\
20 \%\end{array}$ & $\begin{array}{l}\min \\
\min \\
\min \\
\min \end{array}$ \\
\hline & Coûts d'aménagement & $10 \%$ & budget prévisionnel & $100 \%$ & Max \\
\hline
\end{tabular}


Figure 6 Évaluation multicritère du projet de piste cyclable. Municipalité de Lac-Sergent, MRC de Portneuf, 1996

a) Résultats de l'évaluation multicritère

\begin{tabular}{|l|c|c|c|c|c|}
\hline \multicolumn{6}{|c|}{ Con Matrice de concordance } \\
\hline SCEN1 & $\mathbf{1}$ & $\mathbf{0 . 8 4}$ & $\mathbf{0 . 6 3}$ & $\mathbf{0 . 5 3}$ & $\mathbf{0 . 6 4}$ \\
\cline { 2 - 6 } SCEN2 & $\mathbf{0 . 8 3}$ & 1 & $\mathbf{0 . 6 5}$ & 0.57 & $\mathbf{0 . 6 2}$ \\
\cline { 2 - 6 } SCEN3 & $\mathbf{0 . 4}$ & 0.4 & 1 & 0.85 & $\mathbf{0 . 4 5}$ \\
\cline { 2 - 6 } SCEN4 & $\mathbf{0 . 5 2}$ & $\mathbf{0 . 5 3}$ & $\mathbf{0 . 7 7}$ & $\mathbf{1}$ & $\mathbf{0 . 4 4}$ \\
\cline { 2 - 6 } SCEN5 & $\mathbf{0 . 6 4}$ & $\mathbf{0 . 6 5}$ & $\mathbf{0 . 6 9}$ & $\mathbf{0 . 6 9}$ & $\mathbf{1}$ \\
\hline
\end{tabular}

\begin{tabular}{|l|c|c|c|c|c|}
\hline \multicolumn{6}{|c|}{ Matrice des degrés de crédibilité } \\
\hline SCEN1 & $\mathbf{1}$ & $\mathbf{0 . 8 4}$ & $\mathbf{0}$ & $\mathbf{0}$ & $\mathbf{0}$ \\
\cline { 2 - 6 } SCEN1 SCEN2 & SCEN3 & SCEN4 & SCEN5 \\
\hline SCEN2 & $\mathbf{0 . 8 3}$ & 1 & $\mathbf{0}$ & $\mathbf{0}$ & $\mathbf{0}$ \\
\cline { 2 - 6 } SCEN3 & $\mathbf{0}$ & $\mathbf{0}$ & $\mathbf{1}$ & $\mathbf{0}$ & $\mathbf{0}$ \\
\cline { 2 - 6 } SEEN4 & $\mathbf{0}$ & $\mathbf{0}$ & $\mathbf{0 . 7 7}$ & $\mathbf{1}$ & $\mathbf{0}$ \\
\cline { 2 - 6 } SCEN5 & $\mathbf{0}$ & $\mathbf{0}$ & $\mathbf{0}$ & $\mathbf{0}$ & $\mathbf{1}$ \\
\hline
\end{tabular}

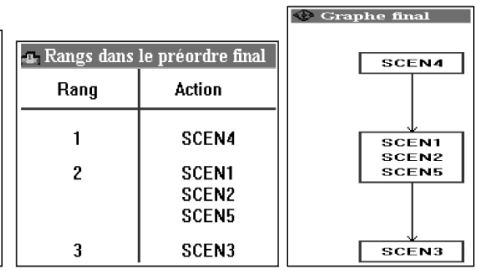

b) Priorité des points de vue du Service d'aménagement et sans veto

\begin{tabular}{|c|c|c|c|c|c|}
\hline \multicolumn{6}{|c|}{ C. Matrice de concordance } \\
\hline & \multicolumn{5}{|l|}{ SCEN } \\
\hline EEN1 & 1 & 0.92 & 0.65 & 0.62 & 0.75 \\
\hline EN2 & 0.79 & & 0.8 & 0.8 & 0.63 \\
\hline SCEN3 & 0.55 & 0.5 & 1 & 0.96 & 0.74 \\
\hline SCEN4 4 & 0.55 & 0.54 & 1 & 1 & 0.74 \\
\hline SCEN5 & 0.69 & 0.66 & 0.77 & 0.77 & 1 \\
\hline
\end{tabular}

\begin{tabular}{|l|c|c|c|c|c|}
\hline Malrice des degrés de crédibilité \\
\hline SCEN1 & 1 & 0.92 & 0.65 & 0.62 & 0.75 \\
\cline { 2 - 6 } SCEN2 & 0.79 & 1 & 0.8 & 0.8 & 0.63 \\
\cline { 2 - 6 } SCEN3 & 0.55 & 0.5 & 1 & 0.96 & 0.74 \\
SCEN4 & 0.55 & 0.54 & 1 & 1 & 0.74 \\
\cline { 2 - 6 } SCEN5 & 0.69 & 0.66 & 0.77 & 0.77 & 1 \\
\hline
\end{tabular}

\begin{tabular}{|c|c|}
\hline \multicolumn{2}{|c|}{ Rangs dans le préordre final } \\
\hline Rang & Action \\
\hline 1 & SCEN2 \\
2 & SCEN1 \\
& SCEN5 \\
3 & SCEN3 \\
& SCEN4 \\
\end{tabular}

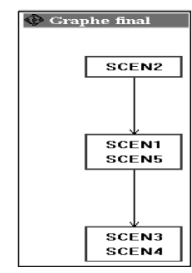

c) Considération unique sur points de vue du Service d'aménagement avec 11 critères prioritaires

\begin{tabular}{|c|c|c|c|c|c|}
\hline \multicolumn{6}{|c|}{ Con Matrice de concordance } \\
\hline$>$ & SCEN1 & $\mathrm{SCEN2}$ & SCEN: & SCEN & SCENS \\
\hline SCEN1 & 1 & 1 & 0.75 & 0.75 & 0.75 \\
\hline SCEN2 & 0.86 & 1 & 0.75 & 0.75 & 0.71 \\
\hline SCEN3 & 0.35 & 0.39 & 1 & 1 & 0.64 \\
\hline SCEN4 4 & 0.35 & 0.39 & 1 & 1 & 0.64 \\
\hline SCEN5 & 0.52 & 0.52 & 1 & 1 & 1 \\
\hline
\end{tabular}

\begin{tabular}{|c|c|c|c|c|c|}
\hline Matrice des degrés de credibilité \\
\hline SCEN1 & $\mathbf{1}$ & $\mathbf{1}$ & $\mathbf{0 . 7 5}$ & $\mathbf{0 . 7 5}$ & $\mathbf{0 . 7 5}$ \\
\hline SCEN 2 & $\mathbf{0 . 8 6}$ & $\mathbf{1}$ & $\mathbf{0 . 7 5}$ & $\mathbf{0 . 7 5}$ & $\mathbf{0 . 7 1}$ \\
\hline SCEN 3 & $\mathbf{0 . 3 5}$ & $\mathbf{0 . 3 9}$ & 1 & 1 & $\mathbf{0 . 6 4}$ \\
SCEN4 & $\mathbf{0 . 3 5}$ & $\mathbf{0 . 3 9}$ & 1 & 1 & $\mathbf{0 . 6 4}$ \\
SCENS5 & $\mathbf{0 . 5 2}$ & $\mathbf{0 . 5 2}$ & 1 & 1 & $\mathbf{1}$ \\
\hline
\end{tabular}

\begin{tabular}{|c|c|}
\hline \multicolumn{2}{|c|}{ Rangs dans le preordre final } \\
Rang & Action \\
\hline 1 & SCEN1 \\
& SCEN2 \\
& SCEN5 \\
2 & SCEN3 \\
& SCEN4 \\
\hline
\end{tabular}

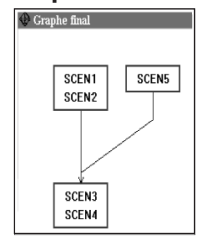

a) matrice de concordance: la concordance exprime la mesure dans laquelle la proposition «scénario a est meilleur que b » est vraie. Elle est représentée sur une échelle de 0 à 1 . ELECTRE III introduit la notion de concordance locale (par paire actions-critères) et de concordance globale (pour l'ensemble des scénarios). La matrice de concordance exprime les résultats globaux pour les scénarios comparés deux à deux. Ces valeurs servent à l'analyste pour orienter les analyses de sensibilité.

b) matrice des degrés de crédibilité: Elle rend compte du degré de surclassement entre les scénarios, au lieu de les accepter ou de les rejeter en bloc. Plus l'indifférence est forte entre deux scénarios, plus cette valeur tend vers 1. Parallèlement, cette valeur s'approche de 0 pour exprimer qu'on tend vers l'incomparabilité. Cette matrice témoigne de la qualité du graphe final et de la matrice de concordance. Ces valeurs sont calculées en tenant compte de tous les critères pour lesquels « b » est strictement préféré à « a », ainsi que des seuils de veto éventuellement spécifiés.

c) graphe final: Ce graphe synthétise l'ensemble des informations fournies par l'analyse multicritère. Dans la figure 6a, il montre que pour l'ensemble des intervenants, le scénario 4 de la firme BPR est le meilleur et le scénario 3 des résidents de la Zone-H s'avère le moins intéressant. Les autres scénarios sont jugés équivalents et de rang intermédiaire.

En vue de faciliter le processus décisionnel, il importe maintenant de porter un jugement sur ces résultats, pour retenir d'autres facteurs d'appréciation, pour réaliser une analyse de sensibilité ou pour effectuer d'autres tests de validation. 


\section{ANALYSE DE SENSIBILITÉ DES RÉSULTATS}

Pour confirmer ces résultats, l'analyse de sensibilité doit indiquer dans quelle mesure cette mise en rang est due à quelques facteurs isolés ou témoigne plutôt de la concordance globale des paramètres subjectifs choisis. Dans ELECTRE III, l'analyse de sensibilité peut être réalisée en faisant varier le poids des critères, les seuils d'indifférence, les seuils de préférence stricte et les seuils de veto. Ce type de démarche est très utile en cas de désaccord entre les parties. En effet, il permet de calculer l'effet marginal sur la décision finale qui est associée à un compromis sur quelques critères ou sur la pondération qui leur est associée. Dans ce sens, c'est à cette étape de l'AMCD que l'on retrouve, pourvu que les parties y consentent, le meilleur potentiel de négociation assistée, une attitude qui est souvent plus difficile à susciter lors d'une réunion de consultation classique. En effet, l'analyse de sensibilité permet d'identifier les vrais enjeux de controverse et comporte tous les outils nécessaires pour détecter les alliances éventuelles entre les groupes d'acteurs (Joerin et al., 2001).

Deux analyses de sensibilité ont été effectuées en collaboration avec l'aménagiste qui avait piloté le dossier pour le Service d'aménagement de la MRC de Portneuf afin de tester cette étape du processus de décision. D'abord, il nous a fait part de ses critiques sur certains des indicateurs que nous avions retenus. Par la suite, il a produit une grille de performances impliquant une révision de la pondération des critères, mais sans veto. Les résultats sont présentés à la figure $6 \mathrm{~b}$. Finalement, l'aménagiste a sélectionné, parmi l'ensemble des 43 indicateurs, les onze qu'il considérait comme essentiels et prioritaires, en spécifiant leur pondération. Avec ce dernier tableau de performances (tableau 4), une ultime évaluation a été effectuée (figure 6c). Dans les commentaires accompagnant les grilles de performance, l'aménagiste a bien pris soin d'indiquer que son appréciation (post-évaluation) des critères a été effectuée en fonction de l'état actuel de la piste cyclable ${ }^{8}$, et que deux ans plus tôt (évaluation ex-ante) ses notes auraient pu être différentes.

\section{Tableau 4 Performances sur les critères estimés prioritaires par l'aménagiste de la MRC (Parc linéaire régional Jacques-Cartier/Portneuf)}

\begin{tabular}{|c|c|c|c|c|c|c|c|c|}
\hline Domaine & Rubriques & Pseudo-critères & Poids & Scénario 1 & Scénario 2 & Scénario 3 & Scénario 4 & Scénario 5 \\
\hline \multirow{4}{*}{ 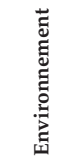 } & \multirow{3}{*}{ Quiétude des résidants } & limitation des accès & $25 \%$ & 3 & 3 & 10 & 10 & 10 \\
\hline & & surveillance du territoire & $25 \%$ & 10 & 10 & 9 & 9 & 9 \\
\hline & & situations non réglementées & $10 \%$ & 7 & 7 & 7 & 7 & 7 \\
\hline & Pouvoir local & retombées économiques & $10 \%$ & 10 & 8 & 1 & 1 & 6 \\
\hline \multirow{7}{*}{ 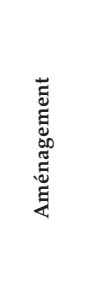 } & Concentration des activités & piste autonome & $4,3 \%$ & 10 & 8 & 5 & 5 & 10 \\
\hline & Topographie & pente prononcée & $4,3 \%$ & 10 & 10 & 3 & 3 & 7 \\
\hline & \multirow{3}{*}{ Sécurité des utilisateurs } & cyclistes avec automobiles & $4,3 \%$ & 10 & 10 & 1 & 1 & 10 \\
\hline & & risques d'accidents & $4,3 \%$ & 10 & 10 & 3 & 3 & 10 \\
\hline & & nombre d'entrées privées & $4,3 \%$ & 10 & 10 & 3 & 3 & 10 \\
\hline & Infrastructures à aménager & largeur de piste inadéquate & $4,3 \%$ & 10 & 10 & 5 & 5 & 8 \\
\hline & Coûts d'aménagement & budget prévisionnel & $4,3 \%$ & 8 & 6 & 3 & 3 & 1 \\
\hline
\end{tabular}




\section{DISCUSSIONS}

Cette application montre l'utilité de la démarche SIG-AMCD pour la planification territoriale. Elle vise à mettre en évidence la pertinence conceptuelle, logistique et scientifique de faciliter la prise de décisions relatives à l'affectation du sol en ayant recours à une combinaison de SIG et d'AMCD. En définitive :

- Le scénario 3 proposé par les citoyens de la Zone-H s'avère le moins intéressant. Il introduit de nouvelles contraintes sans pallier tous les inconvénients des autres scénarios.

- Le scénario 4 se classe habituellement dans le groupe des meilleurs. C'est un scénario qui a été préparé par la firme BPR, laquelle a pris en compte certaines réserves et critiques formulées à l'endroit des autres scénarios. La firme a réussi, dans une bonne mesure, à pallier les inconvénients mentionnés par les résidents.

- Le scénario 2 oscille entre le premier groupe et le groupe médian. Le scénario 5 est plutôt stable dans le groupe médian, alors que le scénario 1 se range surtout entre le groupe médian et le dernier groupe.

- Les scénarios 2, 4 et 5 sont assez distincts entre eux mais constituent, à ce stade, le noyau dur autour duquel pourraient s'articuler les négociations. Pour être considéré, le scénario 5 exigerait des études complémentaires et le scénario 2 est assez sensible à l'effet de veto sur certains critères.

Cet exercice a permis de comparer et d'ordonner les cinq scénarios d'aménagement de la piste cyclable. Les résultats obtenus permettraient d'expliquer aux parties, dans le détail, l'incidence des conditions de site (SIG) et de leurs préférences (SIG et AMCD) sur la pertinence du choix de chacun des tracés.

\section{À propos du classement multicritère des scénarios}

Pour peu que les parties y consentent, les informations disponibles permettraient de négocier sur les critères ainsi que sur leurs pondérations, de manière à mieux définir les conditions des nouvelles affectations des anciennes emprises ferroviaires désaffectées, une amélioration notable par rapport à la situation qui a prévalu dans la MRC de Portneuf (recours aux tribunaux, conflits politiques et amendement de la loi) ${ }^{9}$. Cet exemple d'application a permis de découvrir des tendances communes entre les conséquences d'actions élaborées à partir de points de vue divergents, de même que de comparer les divers scénarios identifiés.

Les deux derniers traitements effectués avec la participation de l'aménagiste ont confirmé les bonnes performances relatives des scénarios 2 et 5 et la faible pertinence du scénario 3 . Ils ont aussi renvoyé au dernier rang le scénario 4 . Bien que ces évaluations reflètent exclusivement les points de vue du Service d'aménagement, les préoccupations des opposants à ce tronçon du parc régional ont été tout de même bien exprimées et prises en compte dans le scénario 4 et ce, même si leur premier choix allait naturellement vers le scénario 3 qui est toujours surclassé par les autres. 
L'AMCD et les SIG éclairent les choix et favorisent le dialogue entre les responsables de la gestion municipale et les citoyens. Bien que le Service d'aménagement de la MRC de Portneuf ait œuvré en toute légalité dans ce dossier, les questions soulevées par les parties ont amené des instances juridiques à questionner la légitimité de la procédure. L'approche proposée ici est compatible avec le cadre juridique existant et pourrait très bien se tenir lors des assemblées de consultation. C'est l'exercice éclairé d'un certain leadership régional qui pourrait réunir les conditions nécessaires pour favoriser leur utilisation afin de résoudre des questions concrètes d'aménagement. Bien que la partie plus technique de la démarche suppose la participation d'experts, ces derniers agissent uniquement comme conseillers pour évaluer les choix et ne sont pas directement impliqués dans la décision qui demeure soumise aux modalités démocratiques usuelles. Grâce à une décomposition détaillée du processus décisionnel, une telle approche peut aider les protagonistes à mieux apprécier la portée concrète de leurs positions (effets sur le territoire) afin de vérifier les limites et les avantages de leurs propositions. L'objectif final consiste à élaborer une ou des interventions consensuelles, bien comprises et bien acceptées par la communauté locale (pour un meilleur exercice de la gouvernance).

\section{Sur la composante SIG}

À cette étape-ci, on pourrait revenir sur la composante SIG. Puisque durant le débat, on distingue bien les aspects physiques des projets et les enjeux sociaux, la maîtrise des données géographiques (SIG) permettrait de rechercher, sur le territoire, d'éventuels nouveaux scénarios d'intervention plus compatibles avec les valeurs et les craintes exprimées par la population. D'un autre côté, dans la recherche du consensus, on pourra distinguer les contraintes spatiales des projets (conditionnés par l'emprise au sol) et les choix dépendant uniquement des préférences des parties à propos desquelles on peut débattre et négocier.

\section{Sur l'analyse de sensibilité}

De plus, l'analyse de sensibilité pourrait amener à une nouvelle modélisation des préférences avec l'assentiment des acteurs. Dans notre étude, on pourrait, par exemple, établir les variantes d'action en dissociant la piste cyclable de la piste de motoneige. Cette distinction pourrait s'accompagner de grilles de critères différentes selon les intérêts (aménagistes, résidents, ou motoneigistes), ce qui permettrait de mieux mettre en évidence ce qui unit et ce qui divise les protagonistes. 


\section{CONCLUSIONS}

Cette étude de cas a montré l'intérêt de l'intégration des SIG et de l'AMCD dans un référentiel d'aménagement du territoire. Il convient de saisir qu'en aménagement, les groupes d'intérêt privilégient souvent des critères particuliers quelquefois antagonistes. Dans une assemblée publique, il est très difficile de trouver une compensation naturelle entre la crainte du "voyeurisme » chez les riverains d'une piste cyclable et les risques liés à la sécurité lors d'une ballade familiale à vélo. La négociation est quasi impossible, parce que les valeurs véhiculées par les parties sont d'ordre émotionnel et n'ont rien de commun. Ainsi, une approche globale considérant à la fois les caractéristiques du territoire, les facteurs techniques d'établissement du projet et l'ensemble des valeurs et craintes de la population a beaucoup plus de chances d'aboutir à un consensus négocié. Néanmoins, le recours aux tribunaux demeure possible en cas de désaccord.

Dans notre exemple, s'il fallait poursuivre les traitements, la prochaine étape consisterait à faire varier les pondérations des critères et le choix des seuils en accord avec toutes les parties concernées par cette planification, avec une intégration plus immédiate du SIG dans les boucles d'itération. La puissance de la procédure décisionnelle provient de l'intégration de la dimension environnementale, des SIG et de l'AMCD dans une démarche qui vise une amélioration des prospectives régionales. Comme nous le posions en introduction, un renforcement des moyens permettant d'optimiser l'évaluation des projets de développement dans un cadre intégré et structuré constitue un des éléments nécessaires pour promouvoir le développement durable.

Toutefois, ce type de traitement nécessite des moyens techniques, du temps et des ressources humaines hautement qualifiées. Bien qu'essentiels au plan technique, ces professionnels ne jouent qu'un rôle complémentaire dans toute la démarche de planification régionale. Leur rôle consiste à favoriser les échanges entre les parties et à orienter certains choix techniques, laissant aux autorités légitimes le soin de négocier l'affectation du territoire. Cette démarche suppose :

- d'une part, l'existence d'une base de connaissance sur le territoire avec les infrastructures humaines et matérielles pour vérifier la disponibilité autant que la fiabilité des données et garantir la pertinence des analyses et des modélisations effectuées; et,

- d'autre part, la volonté chez les acteurs territoriaux d'évaluer la comparabilité de leurs préférences et de les négocier, dans une optique de gouvernance locale.

L'information émerge alors comme un élément décisionnel de premier ordre, et la capacité de la gérer et de l'utiliser à bon escient constituent un apport appréciable des nouvelles technologies d'information pour l'atteinte des objectifs de développement durable, dans un contexte de concurrence globale où la qualité des décisions locales risque de jouer un rôle accru dans les perspectives de développement régional. 
La pertinence de cette combinaison des SIG et de l'AMCD peut s'apprécier à la mesure des défis identifiés à travers l'application de la MRC de Portneuf :

- pour composer en souplesse avec la complexité du cadre légal de la gestion territoriale;

- pour prendre en compte les préoccupations environnementales des citoyens; et,

- pour composer avec les situations où la décision requiert un consensus ou une négociation complexe entre les acteurs concernés.

Cette voie méthodologique pourra s'améliorer avec des contributions visant à poursuivre l'expérimentation avec la participation des autres groupes d'acteurs territoriaux. Les groupes sociaux ont des préférences et des valeurs qui ne sont pas toujours conciliables dans une approche traditionnelle. Dans l'exemple développé ici, la position des motoneigistes est difficilement compatible avec la logique des autres acteurs (résidents et Service d'aménagement). Ce type d'expérimentation devrait tenir compte de manière particulière de ce genre de polarisation des points de vue. Par rapport aux outils utilisés, ce genre d'application justifierait également le développement de solutions logicielles plus ouvertes qui associeraient étroitement, au sein d'une même plate-forme, les composantes du SIG et de l'AMCD. La perspective technologique pourrait se développer également en s'intégrant aux interfaces Internet des municipalités, ou encore en intégrant des composantes d'accès à des entrepôts de données, ce qui permettrait aux citoyens de s'approprier davantage ce type de technologie.

\section{NOTES}

1 Le concept d'état ne doit pas être rapproché ici de celui de situation.

2 La finalité de leur action étant la répartition de la justice sociale et le maintien une certaine pérennité des ressources du milieu au profit des générations futures

3 http ://www.routeverte.com/fr/index.html

4 Représentants des MRC, organismes touristiques, corporations de développement économique, chambre de commerce, Union des producteurs agricoles et clubs de motoneiges.

5 Ce qui peut être considéré comme une participation très importante pour ce genre d'activité et à cette période de l'année (10 juillet).

6 Le jugement prononcé le 8 janvier 1999 par l'Honorable Jean Lemelin j.c.s. a déclaré les règlements 197, 198, 205, et 220 de la MRC de Portneuf nuls et de nullité absolue. Toutefois, le 9 novembre 1999 (Gazette du $1^{\text {er }}$ décembre), l'Assemblée nationale a adopté le projet de Loi 55 modifiant diverses dispositions législatives concernant le domaine municipal. Dans son article 13 (avec effet rétroactif au $1^{\text {er }}$ mai 1993), cette loi a clarifié les pouvoirs des MRC et des Communautés urbaines à l'égard des parcs régionaux et de tout corridor aménagé. Cette loi rend inopérantes les conclusions favorables du jugement du 8 janvier en faveur des villégiateurs et de la ville de Lac-Sergent.

7 Dans le problème décisionnel posé à l'Assemblée de concertation, on ne recherche pas une solution optimale, mais bien à faire un choix parmi les scénarios pour aider le Conseil des représentants de la MRC à promulguer un règlement reflétant un certain consensus. Dans ce contexte, on doit reconnaître : 
- qu'il peut arriver que l'Assemblée de concertation ou le groupe de décideurs ait à départager deux scénarios non comparables et que l'un soit aussi valable que l'autre; et,

- que la préférence soit faible, c'est-à-dire, que la différence relative entre trois actions ne soit pas suffisante pour conclure que si a est meilleure que b et si b est meilleure que c, obligatoirement, a est meilleure que c (classement non transitif).

Dans de telles situations, il faut recourir à une approche par agrégation partielle sur des choix discrets, comme la méthode ELECTRE III, qui permet de comparer les variantes (actions ou scénarios), critère par critère, tout en considérant la non-exclusivité et l'incomparabilité des critères. Cette méthode compare toutes les variantes deux à deux, de manière à produire un surclassement de synthèse en fonction des relations (seuils) de préférence, d'indifférence et d'incomparabilité. Roy (1985) utilise le terme de surclassement pour désigner une situation où une action est préférée à une autre sur la majorité des critères sans lui être totalement supérieure sur tous les critères. Schärlig (1985) argumente de son côté " qu'une action en surclasse une autre si elle est, au moins, aussi bonne que l'autre relativement à une majorité de critères, sans être trop nettement plus mauvaise que cette autre relativement aux autres critères ». Cette approche permet de différencier les solutions efficaces et les solutions acceptables en construisant des ordres partiels (Roy, 1985; Schärlig, 1985). Dans ELECTRE III, les seuils reflètent une estimation des performances en utilisant la logique floue qui permet de considérer des facteurs peu déterminés ou des différences imprécises. Les seuils de discrimination permettent de pallier l'imprécision, l'ambiguïté et l'incertitude associées au signifié d'une mesure.

À la MRC de Portneuf, certains arguments présentés dans le débat pourraient suggérer l'utilisation de vrais critères. Par exemple, la position des résidants à l'égard des motoneigistes suggère l'utilisation d'un vrai critère en vertu duquel toute différence d'appréciation conduirait à ignorer les autres facteurs de décision (Roy et Bouyssou, 1993; Vincke, 1989). Néanmoins, l'utilisation d'un vrai critère nous semble trop rigide. La notion de pseudo-critère ajoute une zone intermédiaire (préférence faible) entre la préférence stricte et l'indifférence, ce qui permet de prendre en compte les hésitations ou les incertitudes des parties. La notion de vrai critère ne nous permettrait pas de tenir compte de l'imprécision entourant l'expression des valeurs des intervenants, telle qu'elle est utilisée.

8 Cette dernière a été effectivement construite en suivant le tracé du scénario 1.

9 Le conflit s'est envenimé lorsque les autres parties ont refusé d'accepter les conclusions des Commissaires de l'Assemblée de consultation ainsi que les règlements (décisions de la MRC) qui en ont découlé.

\section{REMERCIEMENTS}

Cette recherche s'inscrit dans le cadre des travaux de la cellule Géographie du Groupe Écogestion de l'Université Laval et a bénéficié d'un financement du Programme Écorecherche (Plan vert du Canada). Nos remerciements s'adressent également aux membres du Service de l'aménagement du territoire et de l'urbanisme de la MRC de Portneuf pour les nombreux efforts qu'ils ont déployés pour collaborer à cette recherche. 


\section{RÉFÉRENCES BIBLIOGRAPHIQUES}

BRUNET, R., (1997) Le point sur l'aménagement. L'aménagement du territoire en France. Le Dossier, Bimestriel, $\mathrm{n}^{0}$ 7041, juin 1997. La documentation française, Paris, pp. 2-8.

BUOGO, A. et CHEVALLIER, J.-J. (1995) Spatial Information Systems and Information Integration. Computers Environment and Urban Systems, 19 (3) : 161-170.

BURROUGH, P. A and McDONNEL, R. A. (1998) Principles of Geographical Information Systems. New York. Oxford University Press (Coll. «Spatial Information Systems »), $2^{\mathrm{e}} \mathrm{ed}$.

CARON, A. (1995) La prise de décision en urbanisme. Sainte-Foy, Les Publications du Québec, $2^{\mathrm{e}}$ édition revue et augmentée.

CARVER, S. J. (1991) Integrating Multicriteria Evaluation with Geographical Information Systems. International Journal of Geographic Information Science, 5 (3) : 321-339.

CHEVALLIER, J.-J. et DAUDELIN, S. (1996) La géomatique pour l'aide à la décision en gestion des ressources naturelles : exemple de la protection des paysages. Revue internationale de géomatique, 6 (1) : 11-25.

CMED (1992) Agenda 21, Déclaration de Rio. NY, ONU, pages multiples.

DEBRINCAT, L et MEYÈRE, A. (1998) L'aide multicritère à la décision : des potentialités pour l'évaluation des projets de transport collectif en Île-de-France. Metropolis, no 106107 : Évaluer et décider dans les transports, pp. 57-63.

EASTMAN, J. R. (1989) Pushbroom Algorithms for Calculating Distances in Raster Grids. Proceedings, AUTOCARTO 9 : 288-297.

FISHER, M. M. et NIJKAMP, P., dir. (1993) Geographic Information Systems, Spatial Modeling and Policy Evaluation. New York, Spring Verlag.

GIRARD, M., PRÉVIL, C., ROUfFIGNAT, J. et THÉRIAULT, M. (1997) AGRIECOR, Agriculture intensive et écosystèmes régionaux, le SIG du groupe Écogestion pour la région de Portneuf. Écorecherche, Groupe Inter-Conseils, Université Laval.

GOODCHILD, Michael F. (2000) Part 1 - Spatial Analysts and GIS Practitioners. The Current Status of GIS and Spatial Analysis. Journal of Geographical System, 2 (1) : 5-10.

GUAY, P. Y. (2001) Introduction à l'urbanisme: approches théoriques, instruments et critères. Montroyal, Modulo.

GRASLAND, L., dir. (1994) Géopoint 1994. SIG, Analyse spatiale et Aménagement. Groupe Dupont et Université d'Avignon, Avignon.

HANNA, K. S. (2000) The Paradox of Participation and the Hidden Role of Information. A Case Study. American Planning Association Journal, 66 (4) : 398-410.

HICKEY, R. et JANKOWSKI, P. (1997) GIS and Environmental Decision-making to Aid Smelter Reclamation Planning. Environment and Planning, 29 (1) : 5-19.

JACOBS, P. et SADLER, B. (1990) Développement durable et évaluation environnementale: perspectives de planification d'un avenir commun. Conseil canadien de la recherche sur l'évaluation environnementale.

JOERIN, Florent, REY, Marie-Claire, DESTHIEUX, Gilles et NEMBRINI, Aurore (2001) Information et participation pour l'aménagement du territoire. Rôle des instruments d'aide à la décision ». Revue internationale de géomatique, 11 (3-4) : 7-30. 
JOLIVEAU, T. et ETLICHER, B. (1998) Les SIG pour une gestion environnementale des territoires. Éléments de méthodes à partir de deux expériences. Revue internationale de géomatique, 8 (3) : 91-104.

LAARIBI, A. (2000) SIG et analyse multicritère. Paris, Éd. Hermes Science Publications.

MALCZEWSKI, Jacek (1999) GIS and Multicriteria Decision Analysis. New York, John Wiley and Sons, Inc.

MARTIN, N., ST-ONGE, B. et WAAUB, J.-P. (1999) Geographic Tools for Efficient Decision in Watershed Management. Dans Spatial Multicriteria Decision Making and Analysis : A Geographic Information Science Approach, Ashgate Book, ed. by J.-C. Thill, pp. 309-334.

MAYSTRE, L. Y., PICTET, J. et SIMOS, J. (1994) Méthodes multicritères ELECTRE : description, conseils pratiques et cas d'application à la gestion environnementale. Lausanne, Presses polytechniques et universitaires romandes (Coll. " Gérer l'environnement »).

MEREDITH, T. C. (1998) Empowerment, Marginalization, and Public Participation in Community-Based Biodiversity Conservation : Mexican and Canadian Case Studies of Spatial Information Management. Report of Varenius Workshop, Santa Barbara, California.

MOUSSEAU, V. (1993) Problèmes liés à l'évaluation de l'importance relative des critères en aide multicritère à la décision. Paris, Thèse de doctorat, UER Sciences de l'organisation, Université Paris-Dauphine.

MRC DE PORTNEUF (1993) Schéma d'aménagement de la MRC. Service de l'aménagement et de l'urbanisme.

NIJKAMP, P., RIETVELD et VOOGD, H. (1990) Multiple Criteria Evaluation : Issues and Perspectives. Dans Shefer, D., Voogd H. (dir.) Evaluations Methods for Urban and Regional Plans, London, Pion Limited, pp. 147-158.

OPENSHAW, S. and OPENSHAW, C. (1997) Artificial Intelligence in Geography. London, John Willey \& Sons.

PRÉVIL, C. (2000) Approche méthodologique pour la préparation de plans d'aménagement axés sur les préoccupations environnementales. Québec, thèse (Ph.D.) Géographie, Université Laval.

PROULX, M.-U. (1998) La décentralisation gouvernementale. Politique québécoise. L'Action nationale, février 1998, pp. 97-160.

ROUFFIGNAT, J., PRÉVIL, C, MICHAUD, A., GIRARD, M., THÉRIAULT, M. et GUERPILLON, L. (2000) La perception des impacts régionaux et locaux des activités agricoles intensives sur les écosystèmes : une approche régionale. Dans R. Landry et P. Levallois (dir.) Agriculture intensive et écosystème régional, Québec: Presses de l'Université Laval, pp. 7-54.

ROSENHEAD, J. (1989) Rational Analysis of a Problematic World. New York, J. Wiley \& Sons.

ROY, B. (1985) Méthodologie multicritère d'aide à la décision. Paris, Economica.

ROY, B. et BOUYSSOU, D. (1993) Aide multicritère à la décision : méthodes et cas. Paris, Éd. Economica (Coll. « Gestion », Série « Production et techniques quantitatives appliquées à la gestion »). 
SCHÄRLIG, A. (1985) Décider sur plusieurs critères. Panorama de l'aide à la décision multicritère. Lausanne, Éd. Presses Polytechniques Romandes.

(1996) Pratiquer Electre et Prométhée : Un complément à décider sur plusieurs critères. Lausanne, Suisse, Presses polytechniques et universitaires romandes.

SIMON, H. A. (1977) Science of Management Decision. New Jersey, Prentice-Hall.

(1983) Administration et processus de décision. $3^{\mathrm{e}}$ édition (traduction). Paris, Economica, 312 p. [Prix Nobel]

SIMOS, J. (1990) Évaluer l'impact sur l'environnement, une approche originale par l'analyse multicritère et la négociation. Lausanne, Presses Polytechniques et universitaires romandes (Coll. « META »).

THÉRIAULT, M. (1996) SIG : concepts fondamentaux. Québec, Département de Géographie, Université Laval (Série « Notes de cours »).

THÉRIAULT, M., VANDERSMISSEN, M. H., LEE-GOSSELIN, M. et LEROUX, D. (1999) Modelling Commuter Trip Length and Duration Within GIS : Application to an O-D Survey. Journal of Geographic Information and Decision Analysis, 3 (1) : 41-55.

THÉRIAULT, M. et CLARAMUNT, C. (1999) La représentation du temps et des processus dans les SIG : une nécessité pour la recherche interdisciplinaire. Revue internationale de géomatique, 9 (1) : 67-100.

VINCKE, P. (1989) L'aide multicritère à la décision. Bruxelles, Éd. de l’Université de Bruxelles.

VODOZ, L. (1994) La prise de décision par consensus : pourquoi, comment, à quelles conditions. Environnement et Société, (13) : 57-66.

WAAUB, Jean-Philippe (1991) Croissance économique et développement durable : vers un nouveau paradigme du développement. Dans Environnement et développement, questions éthiques et problèmes socio-politiques, Québec, Éd. Fides, sous la dir. de Prades et al., pp. 47-73. 